\title{
Radio observations of candidate magnetic 0 stars
}

\author{
R. S. Schnerr ${ }^{1}$, K. L. J. Rygl ${ }^{1}$, A. J. van der Horst $^{1}$, T. A. Oosterloo ${ }^{2}$, J. C. A. Miller-Jones ${ }^{1}$, \\ H. F. Henrichs ${ }^{1}$, T. A. Th. Spoelstra ${ }^{2}$, and A. R. Foley ${ }^{2}$ \\ 1 Astronomical Institute "Anton Pannekoek", University of Amsterdam, Kruislaan 403, 1098 SJ Amsterdam, The Netherlands \\ e-mail: rschnerr@science.uva.nl \\ 2 ASTRON, 7991 PD, Dwingeloo, The Netherlands
}

Received 25 August 2006 / Accepted 24 May 2007

ABSTRACT

\begin{abstract}
Context. Some O stars are suspected to have to have (weak) magnetic fields because of the observed cyclical variability in their UV wind-lines. However, direct detections of these magnetic fields using optical spectropolarimetry have proven to be very difficult. Aims. Non-thermal radio emission in these objects would most likely be due to synchrotron radiation. As a magnetic field is required for the production of synchrotron radiation, this would be strong evidence for the presence of a magnetic field. Such non-thermal emission has already been observed from the strongly magnetic Ap/Bp stars.

Methods. We have performed 6 and $21 \mathrm{~cm}$ observations using the WSRT and use these, in combination with archival VLA data at $3.6 \mathrm{~cm}$ and results from the literature, to study the radio emission of 5 selected candidate magnetic $\mathrm{O}$ stars.

Results. Out of our five targets, we have detected three: $\xi$ Per, which shows a non-thermal radio spectrum, and $\alpha$ Cam and $\lambda$ Cep, which show no evidence of a non-thermal spectrum. In general we find that the observed free-free (thermal) flux of the stellar wind is lower than expected. This is in agreement with recent findings that the mass-loss rates from $\mathrm{O}$ stars as derived from the $\mathrm{H} \alpha$ line are overestimated because of clumping in the inner part of the stellar wind.
\end{abstract}

Key words. stars: magnetic fields - stars: early-type - stars: general - stars: mass-loss - radio continuum: stars radiation mechanisms: non-thermal

\section{Introduction}

All O-type stars have strong, line-driven winds. They usually have a thermal radio spectrum due to the free-free emission from the ionised stellar wind (Abbott et al. 1980; Bieging et al. 1989; Scuderi et al. 1998). This spectrum can be calculated using:

$$
\begin{aligned}
S_{v}= & 7.26\left(\frac{v}{10 \mathrm{GHz}}\right)^{0.6}\left(\frac{T_{\mathrm{e}}}{10^{4} \mathrm{~K}}\right)^{0.1}\left(\frac{\dot{M}}{10^{-6} M_{\odot} \mathrm{yr}^{-1}}\right)^{4 / 3} \\
& \left(\frac{\mu_{\mathrm{e}} v_{\infty}}{100 \mathrm{~km} \mathrm{~s}^{-1}}\right)^{-4 / 3}\left(\frac{D}{\mathrm{kpc}}\right)^{-2} \mathrm{mJy},
\end{aligned}
$$

(Wright \& Barlow 1975; Panagia \& Felli 1975; Scuderi et al. 1998), where D is the distance to the star, $\dot{M}$ is the mass-loss rate, $T_{\mathrm{e}}$ the electron temperature, $\mu_{\mathrm{e}}$ the mean atomic weight per electron, $v_{\infty}$ the terminal wind velocity and $v$ the observing frequency.

However, about $30 \%$ of the O stars are found to show nonthermal radio emission (see, e.g., Bieging et al. 1989; Drake 1990; Scuderi et al. 1998; Benaglia et al. 2001). This is characterised by a flatter than thermal spectrum, i.e. defined as $\alpha<0.6$, with $S_{v} \propto v^{\alpha}$. White (1985) proposed that synchrotron radiation from the rapidly moving electrons of the wind in a stellar magnetic field could also contribute to the radio emission. This was confirmed by the discovery of non-thermal radio emission in the magnetic Ap/Bp stars (Drake et al. 1987, see also Cassinelli 1984, who report non-thermal emission found in $\sigma$ Ori $\mathrm{E}$ by Churchwell). These stars have strong (of the order of kilogauss), dipole-like magnetic fields. Among O stars only two magnetic stars are known: $\theta^{1}$ Ori C (Donati et al. 2002) and HD 191612
(Donati et al. 2006). Nevertheless, strong indirect evidence exists that many $\mathrm{O}$ stars should have magnetic fields (e.g. Henrichs et al. 2005). One of the main arguments why many $\mathrm{O}$ stars are thought to have (weak) magnetic fields is that their winds show cyclic behaviour on a rotational timescale, which is typically a few days (see Fullerton 2003, for a review). The lack of magnetic field detections is most likely related to the fact that direct measurements of magnetic fields in $\mathrm{O}$ stars are extremely difficult, because of the very few available spectral lines in the optical region. The usual method to measure magnetic fields is to determine the magnetic Zeeman splitting of magnetically sensitive lines with optical spectropolarimetry. The sensitivity of this method decreases towards earlier spectral types, as these stars have fewer spectral lines in the optical region.

The detection of non-thermal radio emission from $\mathrm{O}$ stars with such cyclic variability would be strong evidence that magnetic fields are indeed present in these stars. We have selected five candidate magnetic $\mathrm{O}$ stars that have been studied extensively in the ultraviolet (UV) in order to search for evidence of non-thermal radio emission.

\section{Observations and data reduction}

For this study five targets have been selected from the $10 \mathrm{O}$ stars listed by Kaper et al. (1996), which are the brightest and best studied $\mathrm{O}$ stars in the UV with the International Ultraviolet Explorer (IUE) satellite. All these targets show extensive stellar wind variability, some with well studied cyclic behaviour. The final selection was made on the criteria that the star should be observable with the Westerbork Synthesis Radio Telescope (WSRT) and that the star should have been previously detected 
Table 1. Stellar parameters of the selected O stars. Spectral types are from Walborn $(1973,1976)$. Hipparcos parallaxes were taken from Perryman et al. (1997). All other parameters were taken from the "preferred solution" (some stars have more than one possible set of parameters that fits the observations) of Markova et al. (2004), except for 10 Lac, for which we used Mokiem et al. (2005). We show both the distance from Hipparcos and the distance used for the spectral modeling. As this latter value is used to determine the mass-loss rates, this distance was also used for predicting the thermal radio flux. For the preferred solutions of Markova et al. (2004), we have scaled the distance from the original solution using the absolute magnitudes, as the reddening is assumed to be the same for both solutions.

\begin{tabular}{l|lllll}
\hline \hline & $\xi$ Per & $\alpha$ Cam & 15 Mon & $\lambda$ Cep & 10 Lac \\
\hline HD number & 24912 & 30614 & 47839 & 210839 & 214680 \\
Association/Runaway & Runaway & Runaway & Mon OB1 & Runaway & Lac OB1 \\
Spectral type & O7.5III(n)(f) $)^{a}$ & O9.5 Ia & O7 V ((f)) & O6 I (n)fp & O9 V \\
Parallax (mas) & $1.84 \pm 0.70$ & $0.47 \pm 0.60$ & $3.09 \pm 0.53$ & $1.98 \pm 0.46$ & $3.08 \pm 0.62$ \\
Hip. distance (pc) & $540_{-150}^{+330}$ & $>440$ & $323_{-47}^{+67}$ & $510_{-100}^{+150}$ & $320_{-50}^{+90}$ \\
Spectral mod. distance (pc) & 895 & 765 & 710 & 1089 & 320 \\
Mass $\left(M_{\odot}\right)$ & 52 & 22 & 25 & 58 & 27 \\
Radius $\left(R_{\odot}\right)$ & 25.2 & 19.6 & 9.9 & 23 & 8.3 \\
$T_{\text {eff }}\left(10^{3} \mathrm{~K}\right)$ & 34.0 & 31.0 & 37.5 & 36.2 & 36.0 \\
Luminosity $\left(10^{5} L_{\odot}\right)$ & 7.6 & 3.2 & 1.7 & 8.1 & 1.0 \\
Terminal velocity $\left(\mathrm{km} \mathrm{s}^{-1}\right)$ & 2400 & 1550 & 2200 & 2200 & 1140 \\
Mass-loss $\left(10^{-6} M_{\odot} \mathrm{y}^{-1}\right)$ & $4.0 \pm 1.0$ & $2.9 \pm 0.9$ & $1.2 \pm 0.3$ & $7.7 \pm 2.3$ & $6.1_{-5.5}^{+8.8} \times 10^{-2}$ \\
\hline
\end{tabular}

${ }^{a}$ This is the spectral type given by Walborn (1973), however, a spectral type of O7.5I(n)((f)) was adopted by Markova et al. (2004).

Table 2. Radio detections as a function of wavelength of our targets reported in the literature. When several measurements are available a weighted average is shown; upper limits are $3 \sigma$. Fluxes are from [1] Abbott et al. (1980), [2] Bieging et al. (1989), [3] Drake (1990), [4] Lamers \& Leitherer (1993) and [5] Scuderi et al. (1998).

\begin{tabular}{lcccc}
\hline \hline Star & \multicolumn{4}{c}{ Flux (mJy) } \\
& $2 \mathrm{~cm}$ & $3.6 \mathrm{~cm}$ & $6 \mathrm{~cm}$ & References \\
\hline$\alpha$ Cam & $0.65 \pm 0.13$ & $0.44 \pm 0.04$ & $0.29 \pm 0.04$ & 2,5 \\
15 Mon & $<0.4$ & & $0.40 \pm 0.13$ & 3 \\
& & & $<0.33$ and $<0.18$ & 2,3 \\
$\lambda$ Cep & & $0.38 \pm 0.03$ & $0.40 \pm 0.25$ & 1,4 \\
\hline
\end{tabular}

in the radio region ( $\alpha$ Cam, 15 Mon and $\lambda$ Cep; for $\xi$ Per archive observations from the Very Large Array - VLA - were available). We added 10 Lac because of its brightness and its rich UV history. The stellar parameters of these stars are listed in Table 1.

The radio observations of our targets selected from the literature have been summarised in Table 2. The detection of a $42 \pm 5 \mathrm{mJy}$ source near the optical position of $\xi$ Per by Bohnenstengel \& Wendker (1976) at $11 \mathrm{~cm}(2.7 \mathrm{GHz})$ is discussed in Sect. 3.1.

To complement these measurements, and in order to determine the spectral slopes, we have used WSRT $(6$ and $21 \mathrm{~cm})$ and VLA $(3.6 \mathrm{~cm})$ observations.

We performed observations for all five selected $\mathrm{O}$ stars at $21 \mathrm{~cm}(1.4 \mathrm{GHz})$ with the WSRT during the period from September to November 2005. In addition, 10 Lac was observed at $6 \mathrm{~cm}(4.9 \mathrm{GHz})$. All observations consisted of $12 \mathrm{~h}$ integrations in the Maxi-Short configuration, done in continuum mode with a bandwidth of $8 \times 20 \mathrm{MHz}$. Gain and phase calibrations were done using the calibrator $3 \mathrm{C} 286$, except the observation of $\alpha$ Cam which was calibrated with 3C 48 .

Earlier observations of $\xi$ Per were performed in 1995 at $6 \mathrm{~cm}$ (6 observations in May and June, total of $26 \mathrm{~h}$ ), and $21 \mathrm{~cm}(5 \mathrm{ob}-$ servations in June, July and August, total of $39 \mathrm{~h}$ ). Due to the lower sensitivity of the WSRT at that time, the observations at each frequency were all combined. The $21 \mathrm{~cm}$ observations were calibrated using 3C48 and the $6 \mathrm{~cm}$ observations were calibrated using 3C 48, 3C 147 and 3C 286.
The reduction of the WSRT data was done using the MIRIAD software package.

From the VLA archive we used an X-band $(3.6 \mathrm{~cm}$, $8.5 \mathrm{GHz}$ ) continuum observation taken on 11 Jan. 1999 (program ID AS644-x) of $\xi$ Per. This $0.68 \mathrm{~h}$ observation was taken in $\mathrm{C}$ configuration with a bandwidth of $50 \mathrm{MHz}$. The data were reduced with AIPS, using 3C 48 as a primary and B0411+341 as a secondary calibrator.

\subsection{Distances and mass-loss rates}

As massive stars are relatively far away, their distances as determined by the Hipparcos satellite suffer from systematic errors and and may be underestimated (e.g. Schröder et al. 2004). Distances may also be obtained using the stellar properties derived from spectral modeling. The spectral modeling distance for a star is generally derived from the relation between the spectral type and luminosity, possibly making use of other stars in the same cluster. This distance is used to determine the absolute magnitude of the star and this constrains the stellar radius. As the mass-loss rates determined in this way depend on the radius, we have adopted the distances used in the spectral modeling to calculate the predicted radio fluxes. This ensures that our mass-loss rates and distances are consistent (see also Sect. 4).

\section{Results}

We detected three of our five selected targets (see Table 3). For $\xi$ Per this was the first detection in the radio; all three stars have now been detected for the first time at $21 \mathrm{~cm}$. In general the flux was found to be lower than the predicted thermal flux using Eq. (1) and the stellar parameters from Table 1 (assuming typical values of $\mu_{\mathrm{e}}=1.3$ and $T_{\mathrm{e}}=0.85 T_{\text {eff }}$; Scuderi et al. 1998). Both the predicted thermal flux and a power law fit to the observations are shown in Fig. 1.

We now present the results per source in order of RA.

\section{1. $\xi$ Per}

In the 1995 WSRT observations, $\xi$ Per was not detected at 6 and $21 \mathrm{~cm}$. However, it was detected in the higher $\mathrm{S} / \mathrm{N} 3.6 \mathrm{~cm}$ 
Table 3. Results of our new WSRT and archival VLA observations. The upper limits shown are $5 \sigma$ upper limits.

\begin{tabular}{rllccccc}
\hline \hline $\begin{array}{c}\text { HD } \\
\text { number }\end{array}$ & $\begin{array}{l}\text { star } \\
\text { name }\end{array}$ & $\begin{array}{l}\text { date } \\
(\mathrm{d} / \mathrm{m} / \mathrm{y})\end{array}$ & $\begin{array}{c}\text { freq. } \\
(\mathrm{GHz})\end{array}$ & $\begin{array}{c}\lambda \\
(\mathrm{cm})\end{array}$ & $\begin{array}{c}\text { flux } \\
\mu \mathrm{Jy}\end{array}$ & $\begin{array}{c}\text { spectral } \\
\text { index } \alpha\end{array}$ & array \\
\hline 24912 & $\xi$ Per & $\begin{array}{l}\text { May/Jun./95 } \\
\text { Jun./Aug./95 }\end{array}$ & 4.9 & 6 & $\leq 240$ & & WSRT \\
& & $\begin{array}{l}\text { 11/Jan./99 } \\
\text { 11/4 }\end{array}$ & 8.4 & 3.6 & $\leq 190$ & & WSRT \\
& & 28/Nov./05 & 1.4 & 21 & $100 \pm 30$ & $0.29 \pm 0.14$ & VLA \\
30614 & $\alpha$ Cam & 09/Oct./05 & 1.4 & 21 & $156 \pm 12$ & $0.57 \pm 0.06$ & WSRT \\
47839 & 15 Mon & 13/Oct./05 & 1.4 & 21 & $\leq 250$ & & WSRT \\
210839 & $\lambda$ Cep & 03/Oct./05 & 1.4 & 21 & $98 \pm 21$ & $0.74 \pm 0.11$ & WSRT \\
214680 & 10 Lac & 22/Sep./05 & 4.9 & 6 & $\leq 95$ & & WSRT \\
& & 21/Sep./05 & 1.4 & 21 & $\leq 95$ & & WSRT \\
\hline
\end{tabular}
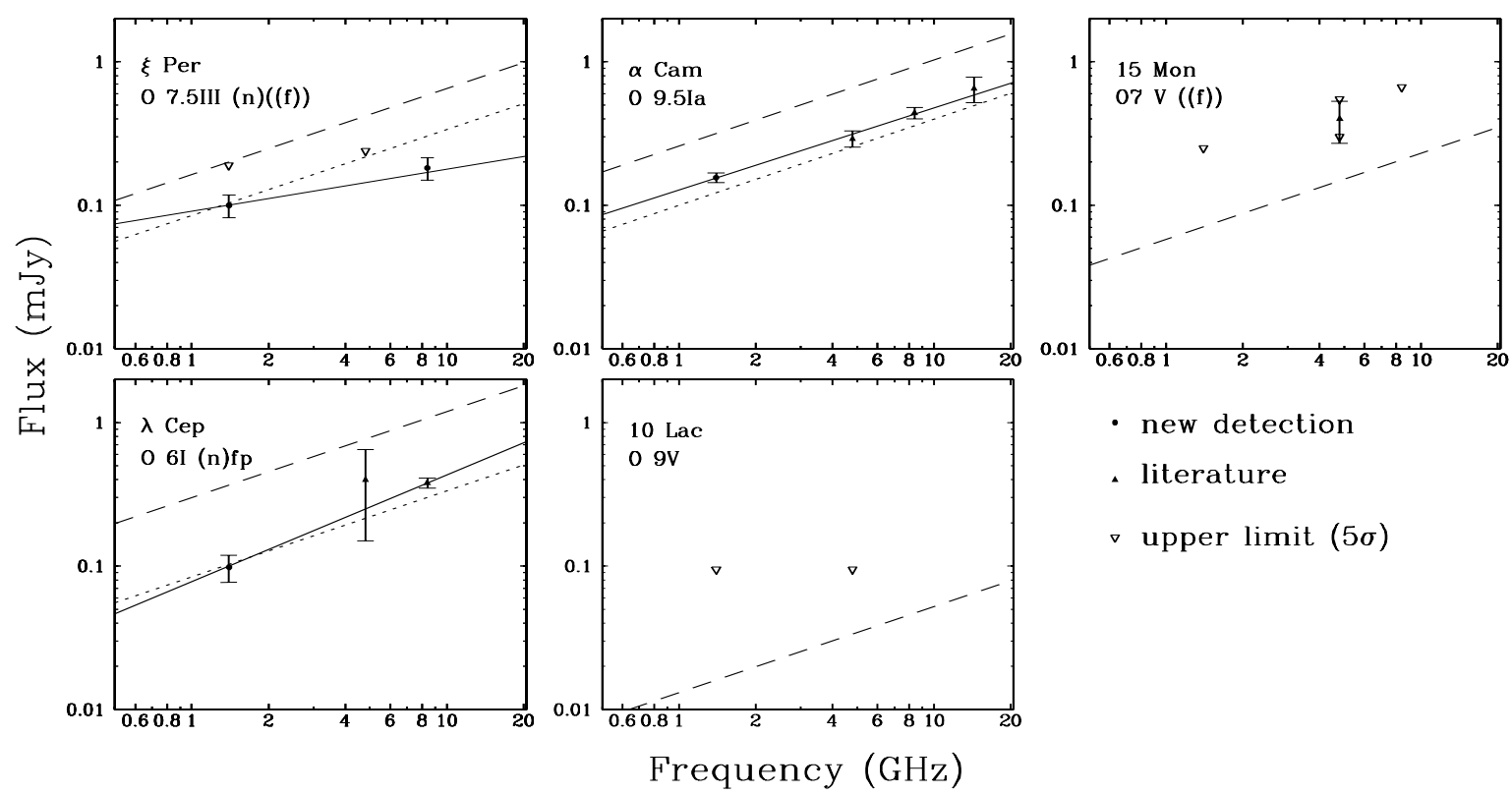

Fig. 1. Radio spectra of the 5 selected targets. Shown are the new results, results taken from the literature, upper limits, the predicted thermal flux (Eq. (1), dashed line), the predicted thermal flux accounting for clumping (Puls et al. 2006, dotted line), and a fit to the observations (solid line). Distances used for estimating the thermal flux are the same as those used for the determination of the mass-loss rates using $\mathrm{H} \alpha$.

(VLA, Jan. 1999) and $21 \mathrm{~cm}$ (WSRT, Nov. 2005) observations. The flux was found to be lower than the predicted thermal flux by a factor of $\sim 2(21 \mathrm{~cm})$ to $\sim 3.5(3.6 \mathrm{~cm})$. The spectrum has a spectral index of $\alpha=0.29 \pm 0.14$, which is lower than the thermal value of 0.6. This is evidence for the presence of a non-thermal contribution to the observed flux.

Puls et al. (2006) found an upper limit for $\xi$ Per of $120 \mu \mathrm{Jy}$ $(3 \sigma)$ from VLA observations on March 9, 2004. As this limit is not consistent with our detection at this wavelength, this might be an indication of variability. To check this, we retrieved the observations from the VLA archive. At the position of $\xi$ Per, we measured a flux density of $154 \pm 39 \mu \mathrm{Jy}$, which, we agree, is not a reliable detection of the source $(3.9 \sigma)$. However, it is consistent with our detection of the source at $169 \pm 30 \mu \mathrm{Jy}$ in 1999 .

Bohnenstengel \& Wendker (1976) detected a source of $42 \pm$ $5 \mathrm{mJy}$ at $2.695 \mathrm{GHz}$ near the optical position of $\xi$ Per. They concluded that this component is either due to an extended $\left(\sim 2^{\prime}\right)$ thermal source of about $10 \mathrm{mJy}$, or to blending of their components A and B (see Fig. 2), in which case they claim that component B has to have a very flat spectrum. As an interferometer such as the WSRT is not very sensitive to extended structures, we cannot exclude the presence of an extended source. We find that component $\mathrm{B}$ has a spectral index of $\alpha \approx-1.1$. It is detected at $5.8 \pm 0.2 \mathrm{mJy}$ at $21 \mathrm{~cm}$, its $6 \mathrm{~cm}$ flux is $1.5 \pm 0.1 \mathrm{mJy}$ and at $3.6 \mathrm{~cm}$ it shows extended structure $\left(10^{\prime \prime} \times 2^{\prime \prime}\right)$ but has very low flux density (peak of $\sim 0.2 \mathrm{mJy} /$ beam). At 3.6 and $6 \mathrm{~cm}$, component A can be resolved into two components with a separation of $12 \pm 1^{\prime \prime}$. The western component has a flux of $6.1 \pm 1.1(6 \mathrm{~cm})$ and $2.6 \pm 0.5 \mathrm{mJy}(3.6 \mathrm{~cm})$ and the eastern component $7.1 \pm 0.9(6 \mathrm{~cm})$ and $3.0 \pm 0.5 \mathrm{mJy}(3.6 \mathrm{~cm})$. In addition we found a third source at

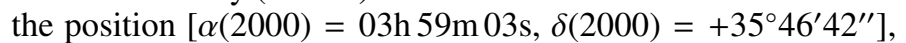
which is not detected at $6 \mathrm{~cm}(\leq 0.24 \mathrm{mJy})$ but is detected at $0.9 \pm 0.2 \mathrm{mJy}$ at $21 \mathrm{~cm}$.

Given the low resolution of the Effelsberg telescope $\left(\sim 5^{\prime}\right.$ at $11 \mathrm{~cm})$ compared to the WSRT $\left(\sim 15^{\prime \prime}\right.$ at $\left.21 \mathrm{~cm}\right)$ and VLA $\left(\sim 2^{\prime \prime}\right.$ at $\left.3.6 \mathrm{~cm}\right)$ observations, we conclude that it is very likely that the source found by Bohnenstengel \& Wendker (1976) is due to blending of all these components.

\section{2. $\alpha$ Cam}

This star shows a thermal spectrum over the entire range from 2 to $21 \mathrm{~cm}$. The flux is found to be lower than the predicted thermal flux by a factor of $\sim 2$.

\subsection{Mon}

Drake (1990) found a $3 \sigma$ upper limit of $400 \mu$ Jy on 24 Jan. 1987 for this source at $2 \mathrm{~cm}$. At $6 \mathrm{~cm}$ Bieging et al. (1989) reported 


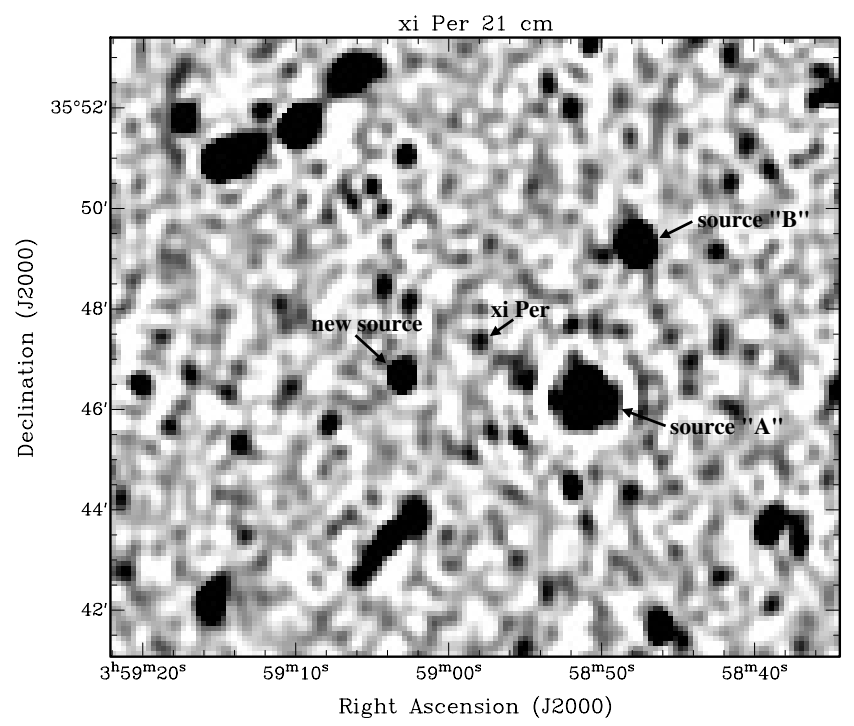

Fig. 2. Our $21 \mathrm{~cm} \xi$ Per observation of 2005. The sources A and B of Bohnenstengel \& Wendker (1976) are indicated, as are $\xi$ Per and a new source that they did not detect (see Sect. 3.1).

a $3 \sigma$ upper limit of $330 \mu \mathrm{Jy}$, and Drake (1990) found an upper limit of $180 \mu \mathrm{Jy}$ on $22 \mathrm{Feb}$. 1986. However, one year later (24 Jan. 1987) Drake (1990) detected the source at the same wavelength at $400 \pm 130 \mu \mathrm{Jy}$. This suggests that the radio flux of 15 Mon is possibly variable.

Due to the unfavourable position, for an E-W array, of 15 Mon on the sky (close to the equator), our $5 \sigma$-upper limit of $250 \mu \mathrm{Jy}$ at $21 \mathrm{~cm}$ is relatively high compared to the expected thermal noise for a $12 \mathrm{~h}$ run.

\section{4. $\lambda$ Cep}

Our detection of $\lambda \mathrm{Cep}$ at $21 \mathrm{~cm}$ allows for an accurate determination of the spectral index. We find that $\lambda$ Cep has an approximately thermal spectrum, with a spectral index of $\alpha=$ $0.74 \pm 0.11$. The flux is, however, a factor of $\sim 3-3.5$ lower than predicted.

\section{5. $10 \mathrm{Lac}$}

We have not detected $10 \mathrm{Lac}$, with a $5 \sigma$ upper limits of $95 \mu \mathrm{Jy}$ at both 21 and $6 \mathrm{~cm}$. This is in agreement with the expected thermal flux based on the determination of the mass-loss rate by Mokiem et al. (2005) of $6.1_{-5.5}^{+8.8} \times 10^{-8} M_{\odot} \mathrm{y}^{-1}$. Our $6 \mathrm{~cm}$ upper limit constrains the mass-loss rate to be lower than $1.3 \times$ $10^{-7} M_{\odot} \mathrm{y}^{-1}$.

\section{The effects of clumping in stellar winds}

As recently discussed by Fullerton et al. (2006) and Puls et al. (2006), it is generally found that mass-loss rates determined from $\mathrm{H} \alpha$ are higher by a factor of $\sim 3-8$ than those derived from radio observations. This is thought to be due to enhanced clumping in the inner part of the wind where the $\mathrm{H} \alpha$ emission originates, compared to the outer part of the wind from which we receive the observed radio emission. As both the $\mathrm{H} \alpha$ and radio emissions are proportional to the density squared, clumping in the wind results in an overestimate of the mass-loss rate. Since we use mass-loss rates derived from $\mathrm{H} \alpha$ to predict the thermal radio fluxes, the fact that the observed fluxes are lower than our predictions is consistent with stronger clumping in the inner part of the wind compared to the outer part.

We have used the same distances as assumed in the $\mathrm{H} \alpha$ modeling. For the $\mathrm{H} \alpha$ modeling the distance (or absolute magnitude) is used to estimate the stellar radius, resulting in $R \propto D$. In these models the mass-loss rate approximately scales with $R$ as $\dot{M} \propto R^{3 / 2}$, which gives $\dot{M} \propto D^{3 / 2}$. Since the predicted radio flux scales as $S_{v} \propto \dot{M}^{4 / 3} D^{-2}$ (Eq. (1)), one finds that the predicted radio flux is approximately independent of the distance.

For $\alpha$ Cam and $\lambda$ Cep, our results are in good agreement with the mass-loss rates determined by Puls et al. (2006), and for $\xi$ Per, the flux observed at $3.6 \mathrm{~cm}$ is in agreement with the upper limits of both possible solutions quoted.

\section{Conclusions}

We have detected three candidate magnetic $\mathrm{O}$ stars at radio wavelengths. Of these three $\xi$ Per shows a non-thermal spectrum and $\alpha$ Cam and $\lambda$ Cep show thermal spectra. As non-thermal radio emission is assumed to be due to synchrotron emission, the detection of a non-thermal radio spectrum in $\xi$ Per strengthens the case that the observed UV line variability observed in this star is caused by a magnetic field.

\subsection{The origin of non-thermal radio emission}

Van Loo et al. (2004, see also Rauw et al. 2002) were able to successfully reproduce the non-thermal emission of the O5 If star Cyg OB2 No. 9, using a model assuming a power-law for the momentum distribution of the relativistic electrons. Reasonable values for the magnetic field that were used are of the order of 10-100 Gauss. However, more recent numerical simulations incorporating individual shocks for the acceleration of electrons (e.g. van Loo et al. 2005; van Loo 2005) suggest that both a magnetic field and a binary companion are required to explain nonthermal radio emission from massive stars. In these simulations, the synchrotron radiation from single massive stars with magnetic fields is produced relatively close to the star, where shocks occur which accelerate the electrons. This radiation is absorbed in the stellar wind due to the large free-free opacity. When a star has a massive binary companion, electrons are accelerated in the wind-wind collision region, where the radio emission can escape because of the lower opacity $\left(\tau_{\text {radio }} \lesssim 1\right)$.

In the less massive strongly magnetic Bp stars, non-thermal radio emission is observed that is thought to be produced by mildly relativistic electrons which are trapped in the stellar magnetosphere (e.g. Drake et al. 1987; Leone et al. 1996). Radio observations of known magnetic massive stars would help to increase our understanding of the relation between magnetic field strength, mass-loss rate and non-thermal radio emission. Unfortunately, the few massive stars that have measured magnetic fields are likely relatively weak radio sources as they have rather weak (order $10^{2}$ Gauss) fields and a low $\dot{M}(\beta$ Cep, V2052 Oph, $\zeta$ Cas and $\tau$ Sco), or are in a crowded field $\left(\theta^{1}\right.$ Ori C).

\section{2. $\xi$ Per, $\lambda$ Cep and $\alpha$ Cam}

$\xi$ Per is a single runaway star (Gies \& Bolton 1986). Runaway stars are stars with high space velocities of $\sim 30-200 \mathrm{~km} \mathrm{~s}^{-1}$ and are usually single stars. Therefore, at least for some single massive stars it is possible to have a (mildly) non-thermal radio spectrum. The detection of variability in the radio flux of close binary 
stars suggests that stellar winds are not as optically thick as generally assumed (Blomme 2005). Due to porosity effects, clumping and asphericity of the mass loss due to magnetic fields, it might be possible to observe radio emission from much closer to the central star. For producing synchrotron emission, a magnetic field is necessary but not sufficient, as relativistic electrons are also required. In the case of $\xi$ Per, and other possibly single massive stars with non-thermal radio spectra, the precise origin of the relativistic electrons needs further investigation. One possible origin in the case of runaway stars would be the bow shock of the star moving through the interstellar material. However, in high resolution radio observations, such as our $3.6 \mathrm{~cm}$ observation of $\xi$ Per, such a bow shock would most likely be resolved.

In principle, variability of the radio flux of $\xi$ Per could change the spectral index since the data at different wavelengths are taken at different epochs. However, observed radio variability of massive stars is usually related to the orbit of a massive companion. Such variability is not expected for $\xi$ Per, but as the mechanism responsible for the relativistic electrons is not completely understood we plan future observations to confirm the non-thermal character of the radio spectrum, and check if variability is present.

The runaway stars $\lambda$ Cep and $\alpha$ Cam, which are presumably single (Gies \& Bolton 1986), have a thermal radio spectrum, but it cannot be excluded that they have a magnetic field. $\lambda$ Cep has a much denser stellar wind, and the non-thermal emission might all be absorbed. In the case of $\alpha \mathrm{Cam}$, the magnetic field might be too weak to produce observable non-thermal emission, but the lack of non-thermal emission could also be due to the location (closer to the star) or strength of the shocks required to produce the relativistic electrons.

Finally, our results agree with recent results by Fullerton et al. (2006) and Puls et al. (2006) that the mass-loss rates as derived from free-free radio emission are significantly lower than those derived from $\mathrm{H} \alpha$ modeling, which is a signature of enhanced clumping in the inner part of stellar wind.

Acknowledgements. We are grateful to J. Puls and M.R. Mokiem for useful discussions on $\mathrm{H} \alpha$ modeling, and to $\mathrm{C}$. Stanghellini for discussions on the radio observations of $\xi$ Per from 2004. This research has made use of the Simbad and ADS databases. The Westerbork Synthesis Radio Telescope is operated by
ASTRON (The Netherlands Foundation for Research in Astronomy) with support from The Netherlands Foundation for Scientific Research NWO. The Very Large Array is part of the National Radio Astronomy Observatory, which is a facility of the National Science Foundation operated under cooperative agreement by Associated Universities, Inc.

\section{References}

Abbott, D. C., Bieging, J. H., Churchwell, E., \& Cassinelli, J. P. 1980, ApJ, 238, 196

Benaglia, P., Cappa, C. E., \& Koribalski, B. S. 2001, A\&A, 372, 952

Bieging, J. H., Abbott, D. C., \& Churchwell, E. B. 1989, ApJ, 340, 518

Blomme, R. 2005, in Proceedings of Massive Stars and High-Energy Emission in OB Associations, ed. G. Rauw, Y. Nazé, \& R. Blomme, 45

Bohnenstengel, H.-D., \& Wendker, H. J. 1976, A\&A, 52, 23

Cassinelli, J. P. 1984, in NASA CP-2358, ed. A. B. Underhill, \& A. G. Michalitisianos, 2

Donati, J.-F., Babel, J., Harries, T. J., et al. 2002, MNRAS, 333, 55

Donati, J.-F., Howarth, I. D., Bouret, J.-C., et al. 2006, MNRAS, 365, L6

Drake, S. A. 1990, AJ, 100, 572

Drake, S. A., Abbott, D. C., Bastian, T. S., et al. 1987, ApJ, 322, 902

Fullerton, A. W. 2003, in Magnetic Fields in O, B and A Stars: Origin and Connection to Pulsation, Rotation and Mass Loss, ASP Conf. Ser., 305, 333 Fullerton, A. W., Massa, D. L., \& Prinja, R. K. 2006, ApJ, 637, 1025

Gies, D. R., \& Bolton, C. T. 1986, ApJS, 61, 419

Henrichs, H. F., Schnerr, R. S., \& ten Kulve, E. 2005, in The Nature and Evolution of Disks Around Hot Stars, ASP Conf. Ser., 337, 114

Kaper, L., Henrichs, H. F., Nichols, J. S., et al. 1996, A\&AS, 116, 257

Lamers, H. J. G. L. M., \& Leitherer, C. 1993, ApJ, 412, 771

Leone, F., Umana, G., \& Trigilio, C. 1996, A\&A, 310, 271

Markova, N., Puls, J., Repolust, T., \& Markov, H. 2004, A\&A, 413, 693

Mokiem, M. R., de Koter, A., Puls, J., et al. 2005, A\&A, 441, 711

Panagia, N., \& Felli, M. 1975, A\&A, 39, 1

Perryman, M. A. C., Lindegren, L., Kovalevsky, J., et al. 1997, A\&A, 323, L49

Puls, J., Markova, N., Scuderi, S., et al. 2006, A\&A, 454, 625

Rauw, G., Blomme, R., Waldron, W. L., et al. 2002, A\&A, 394, 993

Schröder, S. E., Kaper, L., Lamers, H. J. G. L. M., \& Brown, A. G. A. 2004, A\&A, 428, 149

Scuderi, S., Panagia, N., Stanghellini, C., Trigilio, C., \& Umana, G. 1998, A\&A, 332,251

van Loo, S. 2005, in Massive Stars and High-Energy Emission in OB Associations, ed. G. Rauw, Y. Nazé, \& R. Blomme, 61

Van Loo, S., Runacres, M. C., \& Blomme, R. 2004, A\&A, 418, 717

van Loo, S., Runacres, M. C., \& Blomme, R. 2005, A\&A, 433, 313

Walborn, N. R. 1973, AJ, 78, 1067

Walborn, N. R. 1976, ApJ, 205, 419

White, R. L. 1985, ApJ, 289, 698

Wright, A. E., \& Barlow, M. J. 1975, MNRAS, 170, 41 